\title{
Assessment of Undiscovered Oil and Gas Resources of the Williston Basin Province of North Dakota, Montana, and South Dakota, 2008
}

Using a geology-based assessment method, the U.S. Geological Survey estimated mean undiscovered volumes of 3.8 billion barrels of undiscovered oil, 3.7 trillion cubic feet of associated/dissolved natural gas, and 0.2 billion barrels of undiscovered natural gas liquids in the Williston Basin Province, North Dakota, Montana, and South Dakota.

\section{Introduction}

The U.S. Geological Survey (USGS) completed an assessment of the undiscovered oil and gas resources in conventional and continuous accumulations of the Williston Basin Province of North Dakota, eastern Montana, and northwestern South Dakota (fig. 1). The assessment is based on geologic elements of a total petroleum system (TPS) that include (1) source-rock distribution, thickness, organic richness, maturation, petroleum generation, and migration; (2) reservoir-rock type (conventional or continuous), distribution, and quality; and (3) character of traps and time of formation with respect to petroleum generation and migration. Detailed

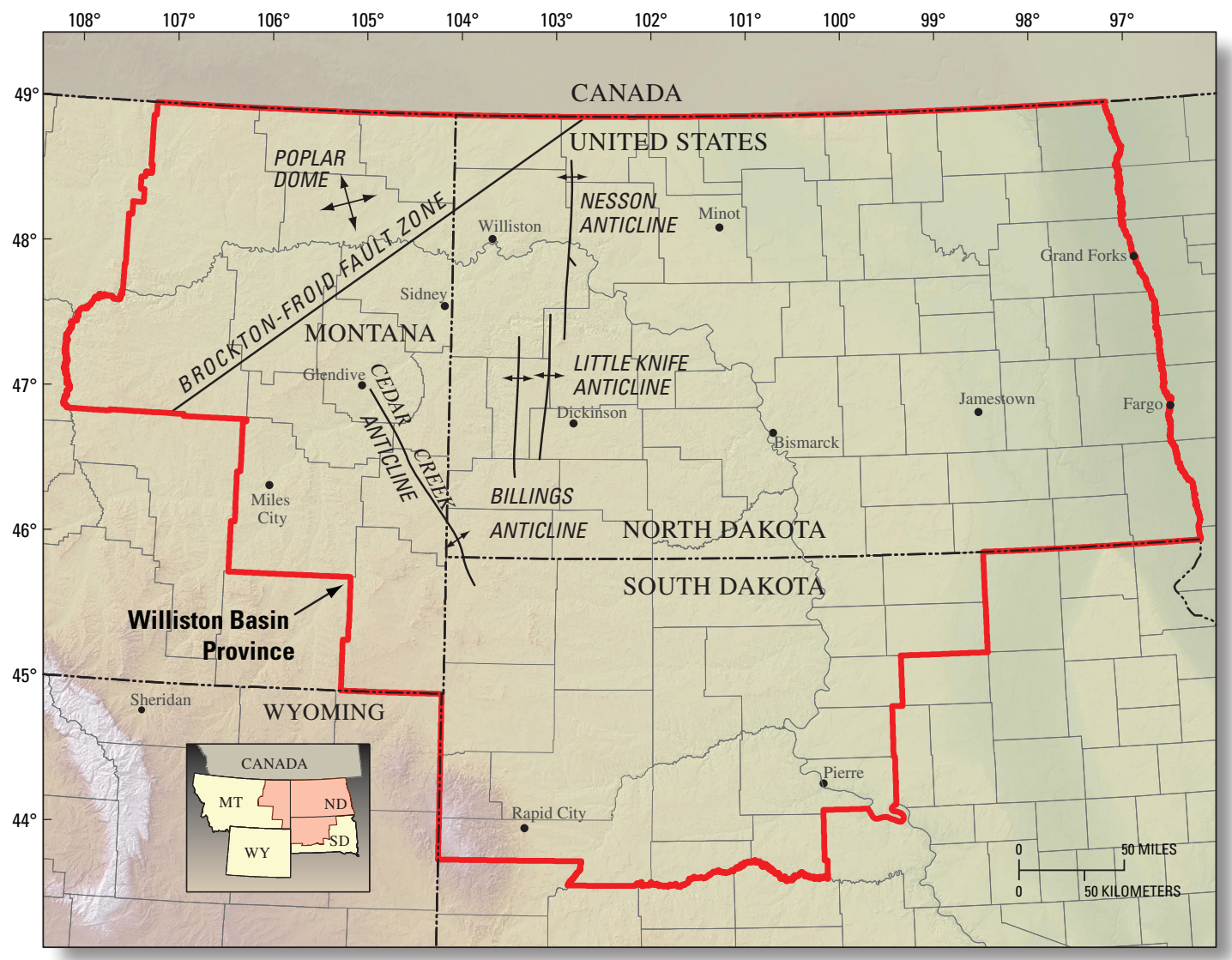

framework studies in stratigraphy and structural geology and the modeling of petroleum geochemistry, combined with historical exploration and production analyses, were used in estimating the undiscovered, technically recoverable oil and gas resources of the entire stratigraphic section in the U.S. part of the basin. Using this framework, the USGS defined 11 TPSs and 19 assessment units (AU) within them, and undiscovered oil and gas resources were quantitatively estimated within each AU (table 1). The assessment of the Bakken Formation is included here, and it was also published previously as USGS Fact Sheet 2008-3021.

\section{Resource Summary}

The USGS estimated means of 3,844 million barrels of oil (MMBO), 3,705 billion cubic feet of gas (BCFG), and 202 million barrels of total natural gas liquids (MMBNGL) for undiscovered continuous and conventional resources in the Williston Basin Province (table 1).

The assessment indicates that most of the undiscovered oil and gas resides within the Bakken Formation as a continuous reservoir with a mean of 3,645 MMBO, whereas undiscovered oil from conventional reservoirs has a mean of 197 MMBO. All of the undiscovered continuous gas resides in the Bakken with a mean of 1,848 BCFG, and in coalbed gas with a mean of 882 BCFG.

Most of the undiscovered conventional oil resides in the Mission Canyon-Charles AU with a mean of $45 \mathrm{MMBO}$, and the Red River Fairway AU with a mean of $30 \mathrm{MMBO}$. The remainder from Paleozoic AUs is estimated to contain a mean of 122 MMBO. Undiscovered conventional gas resources reside in Paleozoic and shallow biogenic gas AUs with a mean of 975 BCFG.

Figure 1. Williston Basin Province of North Dakota, Montana, and South Dakota. 
Table 1. Williston Basin Province assessment results.

[MMBO, million barrels of oil; BCFG, billion cubic feet of gas; MMBNGL, million barrels of natural gas liquids. Results shown are fully risked estimates. For gas accumulations, all liquids are included as NGL (natural gas liquids). F95 represents a 95-percent chance of at least the amount tabulated; other fractiles are defined similarly. TPS, total petroleum system; AU, assessment unit. Gray shading indicates not applicable]

\begin{tabular}{|c|c|c|c|c|c|c|c|c|c|c|c|c|c|}
\hline \multirow{3}{*}{$\begin{array}{l}\text { Total Petroleum System } \\
\text { and Assessment Unit }\end{array}$} & \multirow{3}{*}{\begin{tabular}{|l|} 
Field \\
Type
\end{tabular}} & \multicolumn{12}{|c|}{ Total Undiscovered Resources } \\
\hline & & \multicolumn{4}{|c|}{ Oil (MMBO) } & \multicolumn{4}{|c|}{ Gas (BCFG) } & \multicolumn{4}{|c|}{ NGL (MMBNGL) } \\
\hline & & F95 & $\mathrm{F50}$ & F5 & \begin{tabular}{|l|l} 
Mean \\
\end{tabular} & F95 & $\mathrm{F50}$ & $\mathrm{F} 5_{5}$ & Mean & F95 & F50 & F5 & Mean \\
\hline \multicolumn{14}{|l|}{ Bakken-Lodgepole TPS } \\
\hline Elm Coulee-Billings Nose AU & Oil & 374 & 410 & 450 & 410 & 118 & 198 & 332 & 208 & 8 & 16 & 29 & 17 \\
\hline Central Basin-Poplar Dome AU & Oil & 394 & 482 & 589 & 485 & 134 & 233 & 403 & 246 & 10 & 18 & 35 & 20 \\
\hline $\begin{array}{l}\text { Nesson-Little Knife } \\
\text { Structural AU }\end{array}$ & Oil & 818 & 908 & 1,007 & 909 & 260 & 438 & 738 & 461 & 19 & 34 & 64 & 37 \\
\hline $\begin{array}{l}\text { Eastern Expulsion } \\
\text { Threshold AU }\end{array}$ & Oil & 864 & 971 & 1,091 & 973 & 278 & 469 & 791 & 493 & 20 & 37 & 68 & 39 \\
\hline $\begin{array}{l}\text { Northwest Expulsion } \\
\text { Threshold AU }\end{array}$ & Oil & 613 & 851 & 1,182 & 868 & 224 & 411 & 754 & 440 & 16 & 32 & 64 & 35 \\
\hline \multicolumn{14}{|l|}{ Coalbed Gas TPS } \\
\hline Fort Union Coalbed Gas AU & Gas & & & & & 368 & 791 & 1,701 & 882 & 0 & 0 & 0 & 0 \\
\hline $\begin{array}{l}\text { Total Continuous } \\
\text { Resources }\end{array}$ & & & & & \begin{tabular}{|c|}
3,645 \\
\end{tabular} & & & & 2,730 & & & & 148 \\
\hline \multicolumn{14}{|l|}{ Bakken-Lodgepole TPS } \\
\hline \multirow{2}{*}{ Middle Sandstone Member AU } & Oil & 1 & 4 & 8 & 4 & 0 & 1 & 3 & 2 & 0 & 0 & 0 & 0 \\
\hline & Gas & & & & & 0 & 0 & 0 & 0 & 0 & 0 & 0 & 0 \\
\hline \multirow{2}{*}{ Lodgepole AU } & Oil & 2 & 7 & 18 & 8 & 1 & 4 & 11 & 5 & 0 & 0 & 1 & 0 \\
\hline & Gas & & & & & 0 & 0 & 0 & 0 & 0 & 0 & 0 & 0 \\
\hline \multicolumn{14}{|l|}{ Winnipeg-Deadwood TPS } \\
\hline \multirow{2}{*}{ Winnipeg-Deadwood AU } & Oil & 1 & 4 & 10 & 5 & 3 & 9 & 24 & 11 & 0 & 0 & 1 & 0 \\
\hline & Gas & & & & & 56 & 161 & 358 & 178 & 3 & 8 & 20 & 9 \\
\hline \multicolumn{14}{|l|}{ Red River TPS } \\
\hline \multirow{2}{*}{ Red River Fairway AU } & Oil & 12 & 29 & 51 & 30 & 11 & 28 & 55 & 30 & 1 & 3 & 6 & 3 \\
\hline & Gas & & & & & 58 & 155 & 314 & 167 & 11 & 30 & 67 & 33 \\
\hline \multirow{2}{*}{ Red River East Margin AU } & Oil & 0 & 2 & 4 & 2 & 0 & 0 & 1 & 0 & 0 & 0 & 0 & 0 \\
\hline & Gas & & & & & 0 & 0 & 0 & 0 & 0 & 0 & 0 & 0 \\
\hline \multirow{2}{*}{$\begin{array}{l}\text { Interlake-Stonewall-Stony } \\
\text { Mountain } \mathrm{AU}\end{array}$} & Oil & 9 & 22 & 44 & 24 & 8 & 22 & 47 & 24 & 1 & 2 & 5 & 2 \\
\hline & Gas & & & & & \begin{tabular}{l|l}
0 \\
\end{tabular} & 0 & 0 & 0 & 0 & 0 & 0 & 0 \\
\hline \multicolumn{14}{|l|}{ Winnipegosis TPS } \\
\hline Winninergeic Alt & Oil & 4 & 11 & 22 & 11 & 2 & 6 & 14 & 7 & 0 & 1 & 1 & 1 \\
\hline WVimilegosis AU & Gas & & & & & 0 & 0 & 0 & 0 & 0 & 0 & 0 & 0 \\
\hline Duperow TPS & & & & & & & & & & & & & \\
\hline Dayeon Ray Souric River A & Oil & 2 & 5 & 12 & 6 & 1 & 3 & 6 & 3 & 0 & 0 & 0 & 0 \\
\hline 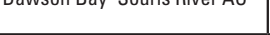 & Gas & & & & & 0 & 0 & 0 & 0 & 0 & 0 & 0 & 0 \\
\hline Dunorene Rivathorat & \begin{tabular}{|l|} 
Oil \\
\end{tabular} & 13 & 26 & 44 & 27 & 9 & 20 & 38 & 22 & 1 & 2 & 4 & 2 \\
\hline 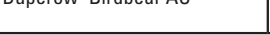 & Gas & & & & & 0 & 0 & 0 & 0 & 0 & 0 & 0 & 0 \\
\hline Cedar Creek Paleozoic Composi & ite TPS & & & & & & & & & & & & \\
\hline Codar Crete Structurel OH & Oil & 6 & \begin{tabular}{l|l|}
19 \\
\end{tabular} & 41 & \begin{tabular}{l|l}
20 & \\
\end{tabular} & \begin{tabular}{l|l}
3 & \\
\end{tabular} & 12 & \begin{tabular}{l|l}
28 \\
\end{tabular} & 13 & 0 & 1 & 2 & 1 \\
\hline edear creek structural AU & Gas & & & & & 0 & 0 & 0 & 0 & 0 & 0 & 0 & 0 \\
\hline Madison TPS & & & & & & & & & & & & & \\
\hline Missinn Canyon-Charles All & Oil & 13 & 43 & 85 & \begin{tabular}{l|l}
45 \\
\end{tabular} & \begin{tabular}{l|l}
9 & \\
\end{tabular} & 33 & 72 & 36 & 1 & 3 & 7 & 3 \\
\hline 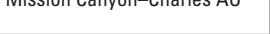 & Gas & & & & & 0 & 0 & 0 & 0 & 0 & 0 & 0 & 0 \\
\hline Tyler TPS & & & & & & & & & & & & & \\
\hline Tulo Condtong & Oil & 4 & 14 & 31 & 15 & 1 & 3 & 7 & 3 & 0 & 0 & 0 & 0 \\
\hline . & Gas & & & & & 0 & 0 & 0 & 0 & 0 & 0 & 0 & 0 \\
\hline Shallow Biogenic Gas TPS & & & & & & & & & & & & & \\
\hline Shallow Biogenic Gas AU & Gas & & & & & 48 & 418 & 1,091 & 475 & 0 & 0 & 0 & 0 \\
\hline $\begin{array}{l}\text { Total Conventional } \\
\text { Resources }\end{array}$ & & & & & 197 & & & & 976 & & & & 54 \\
\hline $\begin{array}{l}\text { Total Undiscovered } \\
\text { Oil and Gas Resources }\end{array}$ & & & & & \begin{tabular}{|l|l|}
3,844 \\
\end{tabular} & & & & 3,705 & & & & 202 \\
\hline
\end{tabular}

\section{For Further Information}

Supporting geologic studies and reports on the assessment method used in the Williston Basin Province assessments of conventional resources are in preparation. Assessment results are available at the USGS Central Energy Team website at URL http:// energy.cr.usgs.gov/oilgas/noga.
Williston Basin Province Assessment Team: Lawrence O. Anna (Task Leader; lanna@usgs.gov), Richard M. Pollastro (Bakken Formation Task Leader), Stephanie B. Gaswirth, Michael D. Lewan, Paul G. Lillis, Laura N.R. Roberts, Christopher J. Schenk, Ronald R. Charpentier, Troy A. Cook, and Timothy R. Klett. 